Article

\title{
Modulation of Campylobacter jejuni Motility, Adhesion to Polystyrene Surfaces, and Invasion of INT407 Cells by Quorum-Sensing Inhibition
}

\author{
Katarina Šimunović ${ }^{1}$, Dina Ramić ${ }^{1}$, Changyun $X u^{2}$ and Sonja Smole Možina ${ }^{1, *(\text { C) }}$ \\ 1 Department of Food Science and Technology, Biotechnical Faculty, University of Ljubljana, Jamnikarjeva 101, \\ 1000 Ljubljana, Slovenia; katarina.simunovic@bf.uni-lj.si (K.S.); dina.ramic@bf.uni-lj.si (D.R.) \\ 2 College of Veterinary Medicine, Iowa State University, 1800 Christensen Dr, Ames, IA 50011, USA; \\ cyxu@iastate.edu \\ * Correspondence: sonja.smole-mozina@bf.uni-lj.si; Tel.: +386-1-320-3751; Fax: +386-1-256-5782
}

Received: 16 December 2019; Accepted: 9 January 2020; Published: 11 January 2020

\begin{abstract}
Campylobacter jejuni is a major foodborne pathogen, and the LuxS-mediated quorum-sensing (QS) system influences its motility, biofilm formation, invasion, host colonization, and virulence. QS therefore represents a target for the control of $C$. jejuni. The aim of this study was to investigate the correlation of QS inhibition with changes in C. jejuni motility, adhesion to polystyrene surfaces, and adhesion to and invasion of INT407 cells. This was achieved by studying (i) the luxS-deficient mutant and (ii) treatment of $C$. jejuni with 20 natural extracts as six essential oils, 11 ethanolic extracts, and three pure compounds. Compared to the wild-type, the $\Delta l u x S$ mutant showed decreased motility, adhesion to polystyrene surfaces, and invasion of INT407 cells. The anti-QS effects of the treatments ( $n=15 / 20)$ were assayed using Vibrio harveyi BB170 bioluminescence. Moderate positive correlation was shown between $C$. jejuni QS reduction and reduced motility $(\tau=0.492, p=0.024)$, adhesion to polystyrene surfaces $(\tau=0.419, p=0.008)$, and invasion $(r=0.394, p=0.068)$. The best overall effect was achieved with a Sedum rosea (roseroot) extract, with $96 \%$ QS reduction, a $1.41 \log (96 \%)$ decrease in adhesion to polystyrene surfaces, and an $82 \%$ decrease in invasion. We show that natural extracts can reduce motility, adhesion to polystyrene surfaces, and invasion of INT407 cells by C. jejuni through modulation of the LuxS (QS) system.
\end{abstract}

Keywords: Campylobacter jejuni; LuxS; quorum-sensing inhibition; AI-2; natural extracts; motility; invasion of INT407 cells; adhesion to polystyrene

\section{Introduction}

Campylobacter jejuni is the leading cause of the most commonly reported bacterial gastroenteritis, campylobacteriosis, worldwide. It is considered an important food-safety hazard, and the major route for its spread is via poultry. Campylobacteriosis manifests as acute watery diarrhea, cramps, and fever, and it is associated with the development of the severe neurological condition known as Guillain-Barre syndrome [1-3]. The number of confirmed campylobacteriosis cases in European countries is more than 250,000 per year, and due to the overuse of antibiotics in veterinary and human medicine, more than $50 \%$ of $C$. jejuni isolates from poultry are now resistant to antibiotics [4]. The risk of resistant C. jejuni spreading through the food chain is therefore high, and thus the acquisition of resistant strains during infection would be more likely. Although campylobacteriosis rarely ends in death, when an infection is caused by a resistant strain, the likelihood of adverse effects increases by $>5$-fold $[2,4,5]$. Altogether, these numbers are alarming, and they represent a major burden for human health and national economies. The estimated total annual cost of campylobacteriosis and its consequences has 
been reported to be $€ 2.4$ billion, and it is likely to rise as the numbers of infections increase $[2,6]$. New targets for $C$. jejuni control and alternative options for its control are thus necessary.

The process of bacterial quorum sensing (QS) is being explored as a novel target for control of pathogens. QS is a form of cell-to-cell communication through small molecules, called autoinducers (AI), through which bacteria regulate their cell responses according to the cell density and are thus related to the behavior of the population as a whole [7,8]. In pathogenic bacteria, QS is often related to their pathogenicity. Examples of this include: Salmonella enterica, where QS controls the expression of virulence-associated genes and thus its virulence in mice [9]; Escherichia coli, where QS regulates motility, biofilm formation, and virulence [10,11]; and Pseudomonas aeruginosa, where QS has a vital role in biofilm formation and development of the disease cystic fibrosis [12].

Quorum sensing is regulated by signaling molecules that accumulate in the bacterial culture-specifically, $\mathrm{N}$-acyl homoserine lactones in Gram-negative bacteria, oligopeptides in Gram-positive bacteria, and a furanosyl borate diester or autoinducer-2 (AI-2) in both Gram-negative and Gram-positive bacteria [8]. In C. jejuni, AI-2-mediated signaling has been described, and the role of the luxS gene in the synthesis of the AI-2 signal was confirmed by Elvers and Park [13]. LuxS is a $S$-ribosylhomociateinase that cleaves a toxic intermediate of the S-adenosylmethionine pathway into homocysteine and 4,5-dihydroxy-2,3-pentanedione, with the latter further spontaneously cyclized into the AI-2 signal molecule $[13,14]$.

The phenotypic changes in the $C$. jejuni luxS-deficient mutant compared to the wild-type include weaker biofilm formation, decreased motility, higher sensitivity to oxidative stress, decreased invasion of Caco-2 cells, decreased virulence in a guinea pig abortion model, and decreased colonization of the chicken intestine [13,15-20]. As the C. jejuni QS-deficient mutant shows critical deficiencies in colonization and virulence, QS inhibition in C. jejuni can be considered a major target for the control of this pathogen in the host.

Bacterial QS can be inhibited by natural extracts and their major pure compounds [8]. For example, garlic extracts reduce Psuedomonas aeruginosa biofilm formation and virulence in vitro and in vivo in a mouse pulmonary infection model [21,22]. Specifically, ajoene is a sulphur-rich compound that was isolated from this garlic extract and is considered to be responsible for this activity. Ajoene shows high anti-QS activity and reduces P. aeruginosa infection in a mouse model, similar to the crude extract [23]. Similarly, rutin is a flavonoid found in various plants, such as olives, buckwheat, and raspberries, and it can reduce biofilm formation and attenuate virulence of pathogenic E. coli through inhibition of QS [24].

Quorum-sensing inhibition by phytochemicals and its consequences in C. jejuni have been poorly studied. It is believed that citrus extracts reduce motility, biofilm formation, invasion, and adhesion of epithelial cells and virulence of $C$. jejuni by modulation of QS $[25,26]$. A Euodia ruticarpa extract has also shown anti-QS activity, although a link between reduction of biofilm formation and QS activity was not shown [27].

Although QS is a prominent target for pathogen control, it is hard to speculate what the effects of its modulation by outside sources would be, such as for phytochemical inhibitors of QS in C. jejuni. The C. jejuni phenotype that is associated with inhibited QS has been described through studies of luxS-deficient mutants, although it is not clear which of its features are caused by lack of cell-to-cell communication and which are caused by disruption of the S-adenosylmethionine metabolic pathway.

The aim of the present study was to determine the influence of QS inhibition on C. jejuni motility, adhesion to polystyrene surfaces, and adhesion to and invasion of INT407 epithelial cells. For this purpose, we chose 20 treatments with essential oils, ethanolic extracts, and pure compounds. The changes in the phenotype of the wild-type C. jejuni 11168 under these treatments were compared to the changes that occurred in the C. jejuni $\Delta l u x S$ mutant to effectively rule out phenotypic changes due to the influence of the treatments on C. jejuni systems other than LuxS. 


\section{Materials and Methods}

\subsection{Bacterial Strains and Growth Conditions}

Campylobacter jejuni NCTC 11168 and C. jejuni 11168 LluxS [19,27] provided by Klančnik A. and Bezek K. (University of Ljubljana) were stored at $-80{ }^{\circ} \mathrm{C}$ in $20 \%$ glycerol and $80 \%$ Mueller-Hinton broth (MHB; Oxoid, UK). They were grown on Mueller-Hinton agar (MHA; Oxoid, UK) at $42{ }^{\circ} \mathrm{C}$ under microaerobic conditions $\left(5 \% \mathrm{O}_{2}, 10 \% \mathrm{CO}_{2}, 85 \% \mathrm{~N}_{2}\right)$ for $24 \mathrm{~h}$. The second passage from each culture was used in the experiments. When necessary, MHA was supplemented with selective medium (SR01176; Oxoid, UK) and growth medium (SR0232E; Oxoid, UK) (MHA-SS) or $30 \mathrm{mg} / \mathrm{L}$ kanamycin (Merck, Darmstadt, Germany). The Vibrio harveyi BB170 reporter strain $[27,28]$ was grown on autoinducer bioassay (AB) medium at $30^{\circ} \mathrm{C}$, which contained $17 \mathrm{~g} / \mathrm{L} \mathrm{NaCl}$ (Merck, Darmstadt, Germany), $12.3 \mathrm{~g} / \mathrm{L}$ $\mathrm{MgSO}_{4}$ (Merck, Darmstadt, Germany), $2 \mathrm{~g} / \mathrm{L}$ casamino acids (BD Bacto, Fisher Scientific, Hampton, $\mathrm{NH}, \mathrm{USA}$ ), $1 \mathrm{mM} \mathrm{K} \mathrm{HPO}_{4}$ (Kemika, Zagreb, Croatia), $0.1 \mathrm{mM}$ L-arginine (Sigma Aldrich, Steinheim am Albuch, Germany), and 1\% (v/v) glycerol (Kemika, Zagreb, Croatia).

\subsection{Essential Oils and Pure Compounds}

All of the essential oils were commercially obtained from companies in Slovenia, as: juniper (Juniperus communis; EO1; from Herbana d.o.o.); oregano (Origanum sp.; EO2); cloves (Syzygium aromaticum; EO3); rosemary (Rosmarinus officinalis; EO4); and thyme (Thymus vulgaris; EO6; all from Lek Veterina d.o.o.); and lavender (Lavandula hybrida; EO5; from M. Jeršek s.p.) (Supplementary Table S1).

The pure compounds used in this study were: carvacrol (P1); rosmarinic acid (P2); and $\gamma$-terpinene (P3), and they were all from Sigma Aldrich (Steinheim am Albuch, Germany) (Supplementary Table S1).

\subsection{Extract Preparation}

Various ethanolic extracts were used in this study for the treatments, and these are described in Supplementary Table S1. These were prepared from the following plants (Kottas Pharma, Vienna, Austria): oregano (Origanum sp.), predistillation (E1) and post-distillation (E2), and from leaves (E3) and flowers (E4) separately; nettle (Urtica dioica), predistillation (E5) and post-distillation (E6); winter savory (Satureja montana) (E7); roseroot (Sedum rosea prev. Rhodiola rosea) (E8); yarrow (Achillea millefolium), predistillation (E9) and post-distillation (E10); and rosemary (Rosmarinus officinalis) (E11).

The plant materials were dried and ground before the ethanolic extractions with $150 \mathrm{~mL} \mathrm{96 \%}$ denatured ethanol (Roth, Germany) added to $20 \mathrm{~g}$ dried material. The suspensions were heated until boiling, stirred for $30 \mathrm{~min}$, and filtered (Rotilabo pleated paper filters; Roth, Germany) to remove undissolved particles. The solvent was evaporated off using a rotary evaporator (Rotavapor; Büchi, Flawil, Switzerland) at $40{ }^{\circ} \mathrm{C}$ and 175 bar. The post-distillation material was prepared with the same protocol but used after the distillation process. Finally, extracts were dried fully using a nitrogen flow and stored at $-20^{\circ} \mathrm{C}$. The roseroot (E8) was provided by Bucar F. (Department of Pharmacognosy, Institute of Pharmaceutical Sciences, University of Graz, Graz, Austria) and was prepared as described by Alperth et al. [29].

\subsection{Determination of Minimal Inhibitory Concentrations}

The minimal inhibitory concentrations (MICs) against C. jejuni of all of the treatments were initially determined using the broth microdilution method, as described previously [30]. For this purpose, all stock solutions were prepared in dimethylsulphoxide (DMSO) at the stock concentration of $40 \mathrm{mg} / \mathrm{mL}$ and then further diluted in MHB. The final concentration of DMSO used in the assays did not exceed $2.5 \%$ in MHB with appropriate DMSO controls also included. Treatments with $0.25 \times$ MICs were chosen for the AI-2 QS inhibition bioassays (Supplementary Table S1). 


\subsection{Autoinducer-2 Bioassay}

To determine the influence of these treatments on C. jejuni QS, AI-2 bioassays were performed. The changes in C. jejuni AI-2 activities or QS were inferred from the changes in the measured luminescence of the $V$. harveyi BB170 reporter strain in relative luminescent units (RLU).

Campylobacter jejuni 11168 and C. jejuni 11168 LluxS [19] cultures in MHB were adjusted to $\mathrm{OD}_{600}$ $0.1\left(5 \times 10^{7} \mathrm{CFU} / \mathrm{mL}\right)$. Here, the treatment stock solutions were prepared as $100 \times$ the concentrations of $0.25 \times$ MIC for each treatment in $100 \%$ DMSO. Then, $50 \mu \mathrm{L}$ of each treatment stock was added to $5 \mathrm{~mL} \mathrm{C}$. jejuni cultures (at $\mathrm{OD}_{600} 0.1$ ) for the final concentration of $0.25 \times \mathrm{MIC}$. Cultures treated with $1 \%$ DMSO were used as controls. The cultures were incubated under microaerobic conditions at $42{ }^{\circ} \mathrm{C}$ for $24 \mathrm{~h}$. To obtain the cell-free supernatants (CFSs) for the following bioassay, samples of $2 \mathrm{~mL}$ were taken and filter sterilized using $0.2 \mu \mathrm{m}$ syringe filters (Sartorius, Göttingen, Germany).

The AI-2 bioassays were performed as previously described $[27,28]$ with some modifications. These QS inhibition bioassays were carried out using the biosensor reporter strain V. harveyi BB170 [27], which was grown for $16 \mathrm{~h}$ at $30{ }^{\circ} \mathrm{C}$ and $150 \mathrm{rpm}$ mixing to approximately $10^{7} \mathrm{CFU} / \mathrm{mL}$ and used at the final concentration of $5 \times 10^{3} \mathrm{CFU} / \mathrm{mL}$ in $\mathrm{AB}$ medium. The C. jejuni CFSs were added to the suspensions of the reporter strain to a final concentration of $10 \%(v / v)$ (i.e., $20 \mu \mathrm{L} \mathrm{CFS} \mathrm{added} \mathrm{to}$ $180 \mu \mathrm{L}$ reporter strain suspension). Sterile medium was used as the blank $[10 \%(v / v) \mathrm{MHB}, 90 \%(v / v)$ $\mathrm{AB}$ medium] and the negative control was 10\% $(v / v)$ MHB and 90\% $(v / v)$ Vibrio harveyi suspension. Kinetic measurements were carried out for the bioluminescence signals of the $V$. harveyi BB170 that were produced as a result of the QS signal that originated from the presence of the CFSs. The relative luminescence signals were measured at $15 \mathrm{~min}$ intervals over $20 \mathrm{~h}$ at $30^{\circ} \mathrm{C}$ in white microtiter plates (Nunc, Thermo Fisher Scientific, Roskilde, Denmark incubated in a microplate reader (Varioskan Lux; Thermo Scientific, Waltham, MA, USA). The relative luminescence signals were interpreted as the QS signal in the C. jejuni CFSs (i.e., higher signals indicated higher concentrations of QS signaling molecules in the CFSs produced by C. jejuni).

When added to MHB, $V$. harveyi produces a background luminescence signal that increases with the concentration of the culture. To find the most stable point of signal production, the CFSs from C. jejuni 11168 $\operatorname{luxS}$, a mutant that cannot produce the quorum-sensing signal AI-2, and fresh MHB were used as the negative controls. The time point when the $V$. harveyi signal production after addition of MHB and C. jejuni 11168 $\operatorname{luxS}$ was still the same but differed from C. jejuni 11168 CFSs (i.e., after $14.5 \mathrm{~h}$ incubation) was used for the calculation of the QS signals attributed to C. jejuni (Supplementary Figure S1).

The experiments were performed as three independent biological replicates and three technical replicates. The data presented are means \pm standard deviation from three biological replicates.

\subsection{Motility Assay}

Campylobacter jejuni 11168 and C. jejuni 11168 LluxS were resuspended in MH broth, and the $\mathrm{OD}_{600}$ was adjusted to 0.1 . The treatments were added to these $C$. jejuni liquid cultures to the final concentrations of $0.25 \times \mathrm{MIC}$ in $1 \%$ DMSO. Control cultures were treated with $1 \%$ DMSO. The prepared cultures were incubated at $42{ }^{\circ} \mathrm{C}$ under microaerobic conditions for $16 \mathrm{~h}$. After these incubations, $1 \mu \mathrm{L}$ of each culture was dropped onto $0.4 \% \mathrm{MH}$ agar and incubated at $42{ }^{\circ} \mathrm{C}$ under microaerophilic conditions for $72 \mathrm{~h}$. The diameter of each colony that formed was measured in $\mathrm{mm}$. The experiments were carried out as three technical replicates, where one plate represented one technical replicate, and in three biological replicates on separate occasions. Data are means \pm standard deviation of the biological replicates. The differences in the colony diameters $(\mathrm{mm})$ were determined, and the effectiveness of the treatments on $C$. jejuni motility inferred from the changes in colony diameters $(\Delta \mathrm{mm})$. 


\subsection{Adhesion to Polystyrene Surfaces}

The treatments were carried out in liquid cultures of C. jejuni 11168 and C. jejuni 11168DluxS (at $\mathrm{OD}_{600}$ 0.1) at the final concentrations of $0.25 \times \mathrm{MIC}$ and 1\% DMSO in MHB. Control cultures were treated with $1 \%$ DMSO. Then, $100 \mu \mathrm{L}$ of the prepared cultures were added to a 96 -well polystyrene microtiter plate (untreated). The plate was incubated at $42{ }^{\circ} \mathrm{C}$ under microaerophilic conditions for $24 \mathrm{~h}$. After the incubation, the wells were washed with phosphate-buffered saline (PBS) three times and treated in an ultrasonic bath (Iskra Pio, Šentjernej, Slovenia) for $10 \mathrm{~min}$. Then, 10-fold serial dilutions were plated onto MH agar. The numbers of adhered C. jejuni were determined as CFU/well. The experiments were carried out in three technical replicates, where one well was one technical replicate, and in three biological replicates on separate occasions. Data are means \pm standard deviation of the biological replicates. The effectiveness of the treatments on C. jejuni adhesion was inferred from the differences between the adhered C. jejuni (CFU/well) of the treated cultures compared to the control.

\subsection{Invasion and Adhesion of INT407 Cells}

The adherence to and invasion of human intestinal epithelial cells by C. jejuni 11168 and C. jejuni $11168 \Delta$ luxS after the treatments were determined using the INT407 cell line (ATCC), according to Negretti and Konkel [31], with some modifications. The effectiveness of the treatments for C. jejuni QS inhibition was inferred from the change in adhered/invaded C. jejuni (CFU/mL) in the INT407 cell cultures compared to the untreated control.

The INT407 cells were cultured in Dulbecco's modified Eagle's medium (DMEM; Corning, Mediatech Inc., Manassas, NA, USA) supplemented with 1\% HEPES (Gipco, Life Technologies, Grand Island, NY, USA), 1\% glutamax (Gipco, Life Technologies, Grand Island, NY, USA), and 10\% fetal bovine serum (FBS) (Sigma, Darmstadt, Germany) in $75 \mathrm{~cm}^{2}$ culture flasks (Corning, Corning, NY, USA) at $37^{\circ} \mathrm{C}$ in $5 \% \mathrm{CO}_{2}$. The INT407 cells were trypsinized ( $0.25 \%$ trypsin; Corning, Corning, VA, USA) and adjusted to $1.5 \times 10^{5}$ cells/mL. One milliliter of the cell suspensions was added to each well of a 24-well microtiter plate (Corning, Corning, NY, USA) and left overnight at $37^{\circ} \mathrm{C}$ under $5 \% \mathrm{CO}_{2}$.

For the bacterial culture preparation, C. jejuni 11168 and C. jejuni 11168 LluxS were suspended in $\mathrm{MH}$ broth, and the $\mathrm{OD}_{600}$ was adjusted to 0.1. Extracts were added to liquid cultures at the final concentration of $0.25 \times \mathrm{MIC}$ in $1 \%$ DMSO. Control cultures were treated with $1 \%$ DMSO. The cultures were then incubated at $42{ }^{\circ} \mathrm{C}$ under microaerobic conditions for $16 \mathrm{~h}$. After the incubations, the cultures were centrifuged $\left(6000 \times g, 20^{\circ} \mathrm{C}\right)$ and washed twice with PBS. The final cultures were resuspended in DMEM1\% (DMEM with 1\% fetal bovine serum, 1\% HEPES, $1 \%$ glutamax), and the OD 600 adjusted to 0.3 . These suspensions were then diluted 1:10 in DMEM1\% to reach an $\mathrm{OD}_{600}$ of 0.03 or $3 \times 10^{7}$ CFU/mL and were then used to infect the INT407 cells.

The plates with the INT407 cells were washed twice with DMEM1\%, inoculated with $1 \mathrm{~mL}$ of the prepared $\mathrm{C}$. jejuni suspensions, and incubated $3 \mathrm{~h}$ at $37^{\circ} \mathrm{C}$ under $5 \% \mathrm{CO}_{2}$. For determination of the total C. jejuni, the plates were washed three times with PBS, and the INT407 cells were lysed with $200 \mu \mathrm{L} 0.1 \%$ Triton X-100 (Sigma, MD, USA) for $5 \mathrm{~min}$ at $37^{\circ} \mathrm{C}$. Following the incubation, $800 \mu \mathrm{L}$ PBS was added to each well, and to determine the CFU/mL of C. jejuni, 10-fold serial dilutions were performed with plating onto MHA plates and incubation at $42{ }^{\circ} \mathrm{C}$ under microaerobic conditions.

For determination of the internalized C. jejuni, the plates were washed twice with DMEM1\%, and $1 \mathrm{~mL}$ DMEM1\% supplemented with $100 \mathrm{mg} / \mathrm{L}$ gentamycin (Gipco, Life Technologies, Grand Island, NY, USA) was added. The plates were incubated for an additional $3 \mathrm{~h}$ at $37^{\circ} \mathrm{C}$ in $5 \% \mathrm{CO}_{2}$. The plates were then washed, the INT407 cells lysed, and the numbers of internalized C. jejuni determined as described above. The number of adhered cells was determined by subtracting the number of internalized cells from the total C. jejuni concentration. The concentrations of the adhered and the internalized C. jejuni are reported as $\log _{10} \mathrm{CFU} / \mathrm{mL}$.

The experiments were carried out in three technical replicates, where one replicate was one well in the plate, and in three biological replicates on separate occasions. Data are means \pm standard deviation of the biological replicates. 


\subsection{Statistical Analysis}

For all the data, before statistical tests were performed, normality was tested with Shapiro-Wilk and Kolmogorov-Smirnov tests. All the CFU/mL and the RLU values were log transformed before analysis. To determine the statistical significance between treated and control cultures, Student's t-tests or Mann-Whitney U-tests were performed, depending on the normality distribution of the data. For correlation analysis, only mean values and not replicates were used for each treatment. Correlations between quorum sensing (luminescence), invasion and adhesion $\left(\log _{10} \mathrm{CFU} / \mathrm{mL}\right)$, motility (diameter), and adhesion to polystyrene surfaces $\left(\log _{10}\right.$ CFU/well) were tested using Pearson's or Kendall's Tau correlation tests, depending on the normality distribution of the data.

All the analyses were performed using the SPSS software, version 22 (IBM Corp., Armonk, NY, USA).

\section{Results}

\subsection{Comparison of Wild-Type C. jejuni 11168 with the luxS-Deficient Mutant}

To evaluate the influence of QS inhibition caused by the loss of the luxS gene that is responsible for AI-2 signal production in C. jejuni, wild-type C. jejuni 11168 and the luxS-deficient mutant were tested for motility on soft agar, adhesion to polystyrene surfaces, and adhesion to and invasion of INT407 cells.

Compared to the wild-type C. jejuni 11168, the C. jejuni $11168 \Delta l u x S$ mutant strain showed a small but significant decrease in motility of $4.7 \%(3.67 \mathrm{~mm} ; p=0.018)$ and significantly lower adherence to polystyrene surfaces of $98.8 \%(1.82-\log$ units; $p<0.001)$, and although adhesion on INT407 cells was not significantly altered, the invasion of the INT407 cells by the C. jejuni 11168 LluxS mutant was significantly lower than for the wild-type by $1.10 \log$ units $(92.1 \% ; p=0.003)$ (Figure 1$)$.

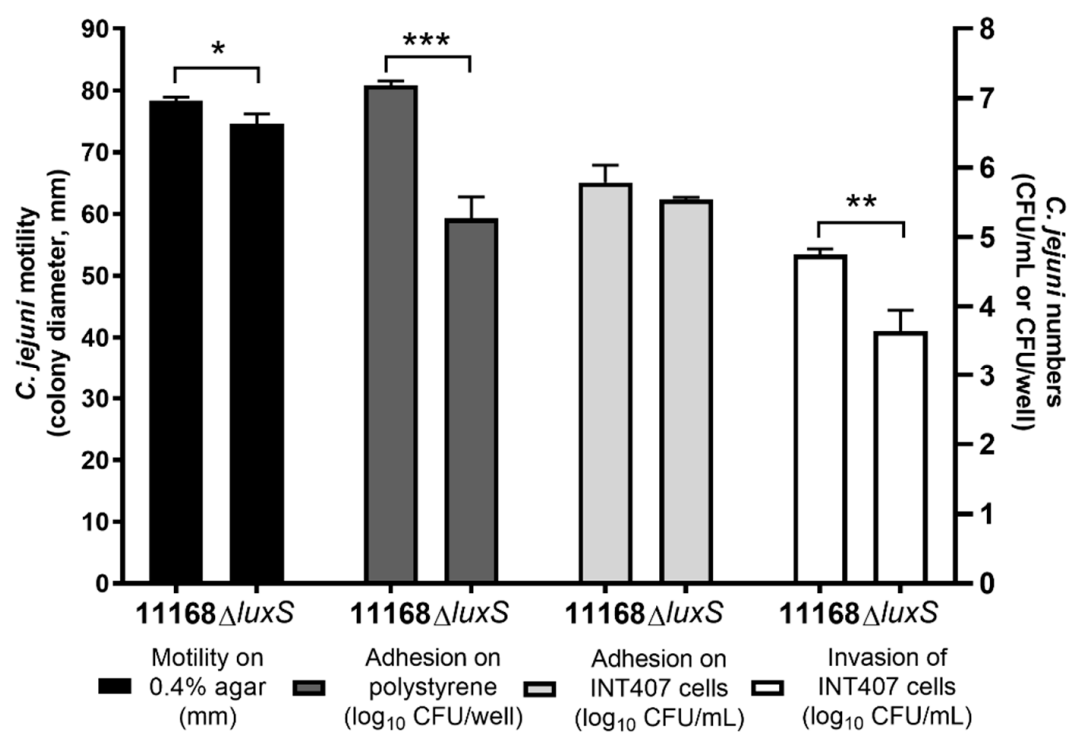

Figure 1. C. jejuni 11168 and C. jejuni $11168 \Delta l u x S$ motility presented as colony diameters (mm), adhesion on polystyrene surfaces presented as $\log _{10} \mathrm{CFU} /$ well, and adhesion to and invasion of INT407 cells presented as $\log _{10} \mathrm{CFU} / \mathrm{mL}$. Data are means \pm standard deviation. ${ }^{*} p<0.05 ;{ }^{* *} p<0.01 ;{ }^{* *} p<0.001$, $\Delta l u x S$ versus 11168 control (Student's $t$-tests).

\subsection{Inhibition of Quorum Sensing of C. jejuni with Essential Oils, Ethanolic Extracts, and Pure Compounds}

Inhibition of C. jejuni QS was examined using the AI-2 bioluminescence assays, where the C. jejuni CFSs, which contained the QS signals, were added to the biosensory strain $V$. harveyi BB170. Reduction of C. jejuni QS by these essential oils, ethanolic extracts, and pure compounds was seen as a decrease in the luminescence signal where the CFSs of treated cultures were added in comparison to the control CFS. 
Most of these treatments $(n=15 / 20)$ significantly lowered the C. jejuni QS signals compared to the control (Figure 2). The luminescence signal decreases ranged from $27 \%$ to $96 \%$ (Table 1 ) with the strongest effect achieved by the ethanolic extract E8 (roseroot). One of the essential oils (EO2, oregano), two of the ethanolic extracts (E1, oregano, predistilled; E11, rosemary), and two of the pure compounds (P1, carvacrol; P2, rosmarinic acid) did not have any significant impacts on C. jejuni QS.

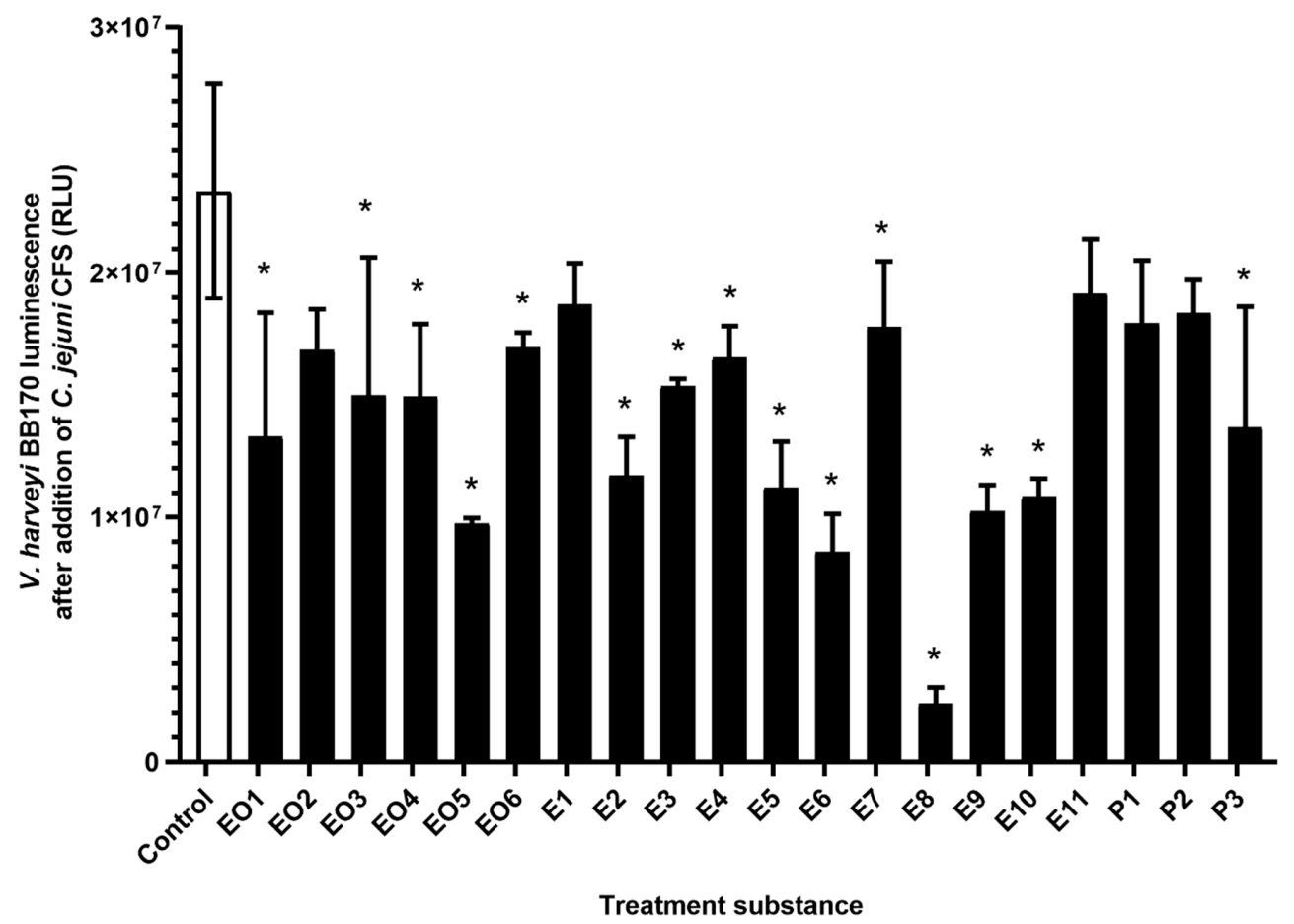

Figure 2. Luminescence of $V$. harveyi BB170 cells (relative fluorescent units; RLU) after addition of C. jejuni 11168 cell-free supernatants from untreated C. jejuni cultures (control, empty bar) and C. jejuni cultures treated with essential oils (EO1-6), ethanolic extracts (E1-11), and pure compounds (P1-3) (full bars). Data are means \pm standard deviation. ${ }^{*}, p<0.05$ versus control (Student's $t$-tests).

Table 1. Inhibition of C. jejuni 11168 QS, motility, adhesion to polystyrene surfaces, and adhesion to and invasion of INT407 cells, as calculated from the cultures treated with essential oils (EO1-6), ethanolic extracts (E1-11), and pure compounds (P1-3) compared to the control, presented as \%inhibition \pm standard deviation.

\begin{tabular}{ccccc}
\hline Treatment & \multicolumn{3}{c}{ Inhibition (\%) for Treated Cultures Compared to Control Culture } \\
\hline Code & QS & Motility & Adhesion to Polystyrene Surfaces & Invasion of INT407 Cells \\
\hline EO1 & $51 \pm 11.6$ & $6 \pm 0.1$ & $99 \pm 0.9$ & $76 \pm 4.3$ \\
EO2 & $37 \pm 1.7$ & $16 \pm 2.0$ & $-10 \pm 0.1$ & $76 \pm 4.5$ \\
EO3 & $44 \pm 9.4$ & $15 \pm 0.8$ & $79 \pm 1.6$ & $76 \pm 2.0$ \\
EO4 & $44 \pm 4.9$ & $19 \pm 2.4$ & $91 \pm 3.8$ & $78 \pm 3.1$ \\
EO5 & $66 \pm 0.7$ & $14 \pm 1.0$ & $96 \pm 1.2$ & $88 \pm 5.6$ \\
EO6 & $36 \pm 0.7$ & $7 \pm 0.6$ & $56 \pm 3.2$ & $88 \pm 7.3$ \\
\hline E1 & $29 \pm 1.3$ & $7 \pm 0.3$ & $13 \pm 0.1$ & $84 \pm 9.8$ \\
E2 & $58 \pm 4.5$ & $11 \pm 0.8$ & $26 \pm 0.1$ & $86 \pm 8.5$ \\
E3 & $43 \pm 0.4$ & $11 \pm 1.4$ & $54 \pm 1.4$ & $85 \pm 7.0$ \\
E4 & $38 \pm 1.5$ & $11 \pm 0.2$ & $36 \pm 0.4$ & $84 \pm 9.8$ \\
E5 & $60 \pm 5.2$ & $9 \pm 0.7$ & $11 \pm 0.4$ & $73 \pm 7.9$ \\
E6 & $71 \pm 6.9$ & $8 \pm 0.2$ & $-1 \pm 0.1$ & $87 \pm 10.2$ \\
E7 & $33 \pm 2.8$ & $9 \pm 0.6$ & $15 \pm 0.3$ & $82 \pm 7.1$ \\
E8 & $96 \pm 15.7$ & $35 \pm 7.6$ & $96 \pm 3.7$ & $90 \pm 8.8$ \\
E9 & $64 \pm 3.5$ & $3 \pm 0.1$ & $57 \pm 3.2$ & $82 \pm 7.1$ \\
E10 & $61 \pm 2.3$ & $5 \pm 0.5$ & $62 \pm 2.9$ & $90 \pm 6.3$ \\
E11 & $27 \pm 1.5$ & $18 \pm 3.8$ & $-18 \pm 0.3$ &
\end{tabular}


Table 1. Cont.

\begin{tabular}{ccccc}
\hline Treatment & \multicolumn{4}{c}{ Inhibition (\%) for Treated Cultures Compared to Control Culture } \\
\hline Code & QS & Motility & Adhesion to Polystyrene Surfaces & Invasion of INT407 Cells \\
\hline P1 & $32 \pm 2.3$ & $6 \pm 0.6$ & $44 \pm 1.6$ & $91 \pm 8.2$ \\
P2 & $31 \pm 1.2$ & $9 \pm 0.3$ & $2 \pm 0.1$ & $73 \pm 4.0$ \\
P3 & $50 \pm 10.4$ & $13 \pm 0.6$ & $91 \pm 0.9$ & $79 \pm 4.2$ \\
\hline
\end{tabular}

\subsection{Modulation of C. jejuni Motility}

The C. jejuni 11168 and the C. jejuni 11168 $\operatorname{luxS}$ motilities were examined in cultures on soft agar that was treated with these essential oils, ethanolic extracts, and pure compounds. The motility of the C. jejuni wild-type was significantly reduced by three of the essential oils (EO1, juniper; EO3, cloves; EO5, lavender), three of the ethanolic extracts (E4, oregano, flowers; E6, nettle, post-distillation; E8, roseroot), and two of the pure compounds (P2, rosmarinic acid; P3, $\gamma$-terpinene). The E8 treatment ( $S$. rosea, roseroot) had the strongest effect, as it decreased the C. jejuni 11168 colonies by $27 \mathrm{~mm}(35 \%)$ (Figure 3A). Although statistical significance was not reached due to the high variability between the experiments here, the motility appeared to be decreased by $>15 \%$ also by essential oils EO2 (oregano) and EO4 (rosemary) and by ethanolic extract E11 (rosemary) (Figure 3A, Table 1, Table S2).
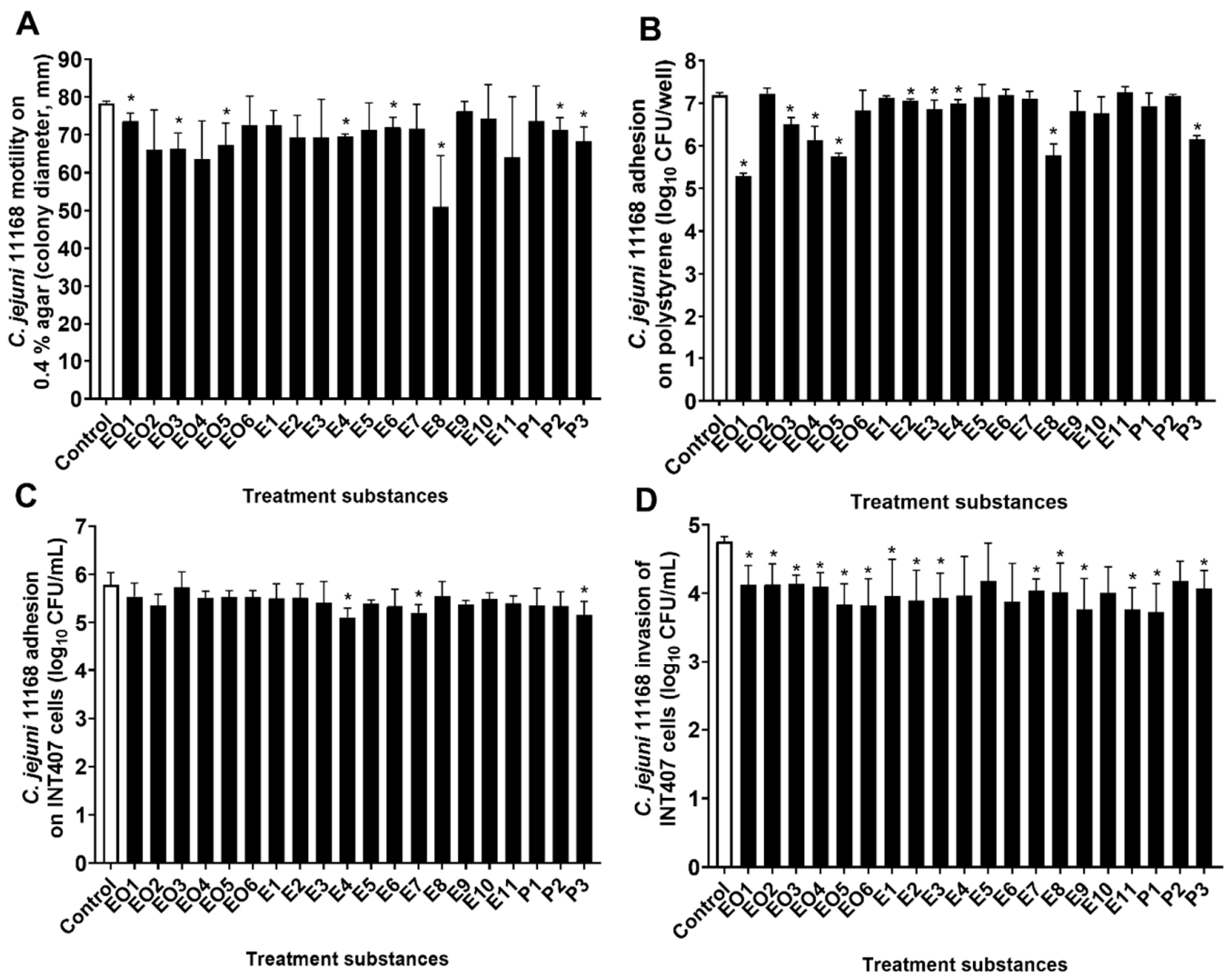

Figure 3. C. jejuni 11168 motility on $0.4 \%$ agar (A); colony diameter (mm), adhesion to polystyrene surfaces (B); $\log 10$ CFU/well, adhesion to (C) and invasion of (D) INT407 cells (CFU/mL) without (control, empty bars) and with treatments with essential oils (EO1-6), ethanolic extracts (E1-11), and pure compounds (P1-3) (full bars). Data are means \pm standard deviations. ${ }^{*} p<0.05$ versus control.

Unlike the wild-type, the motility of the $\Delta l u x S$ mutant strain was not significantly changed by the treatments compared to the control; overall, none of these treatments showed any reduction of the motility of C. jejuni 11168 luxS by $>10 \%$ (Supplementary Figure S2A). 


\subsection{Modulation of C. jejuni Adhesion to Polystyrene Surfaces}

The number of cultivable C. jejuni 11168 and C. jejuni $11168 \Delta l u x S$ that were adhered to a polystyrene surface after a $24 \mathrm{~h}$ incubation with or without these treatments with essential oils, ethanolic extracts, and pure compounds (at $0.25 \times \mathrm{MIC}$ ) were determined as a measure of the C. jejuni biofilm formation on polystyrene surfaces. The adhesion of the wild-type was significantly reduced by nine of these treatments $(n=9 / 20)$ : four essential oils (EO1, juniper; EO3, cloves; EO4, rosemary; EO5, lavender); four ethanolic extracts (E2, oregano, post-distilled; E3, oregano, leaves; E4, oregano, flowers; E8, roseroot); and one of the pure compounds (P3, $\gamma$-terpinene). The reductions caused by the treatments ranged from 0.13 to 1.89 mean $\log$ units (26\% to 99\%) (Figure 3B, Table 1, Table S2), where the greatest reduction was achieved by essential oil EO1, from juniper. The treatments showed no significant effect on C. jejuni 11168 $\operatorname{luxS}$ adhesion to polystyrene surfaces (Figure S2B).

\subsection{Modulation of Adhesion to and Invasion of INT407 Cells by C. jejuni 11168}

The effects of these treatments of C. jejuni 11168 and $11168 \Delta l u x S$ on their adhesion to and invasion of INT407 cells was investigated. C. jejuni cultures were incubated together with these treatments (at $0.25 \times \mathrm{MIC}$ ) for $16 \mathrm{~h}$, then washed and added to the INT407 epithelial cells. In this way, only treated C. jejuni were added without transfer of essential oils, ethanolic extracts, or pure compounds to the INT407 cells. Thus, the changes in adhesion or invasion can be considered to result from phenotypic changes of the C. jejuni cultures due to these treatments.

The adhesion of the treated C. jejuni 11168 cultures to the INT407 cells did not significantly differ from the control for the majority of these treatments $(n=17 / 20)$. Indeed, only treatments E4 (oregano, flowers), E7 (winter savory), and P3 ( $\gamma$-terpinene) showed significantly lower C. jejuni adhesion by 0.67 , 0.58 , and 0.62 mean log units, respectively (Figure 3C, Table S2). The C. jejuni 11168 1 luxS adhesion to INT407 cells was not influenced by any of these treatments (Supplementary Figure S2C).

The invasion of the INT407 cells by $C$. jejuni 11168 was, on the other hand, significantly modified by most of these treatments $(n=15 / 20$; Figure $3 \mathrm{D})$. The reduced invasion ranged from 0.57 to 1.02 mean $\log$ units, with P1 (carvacrol) showing the greatest effect (91\%). The five of these 20 treatments that had no significant effects on invasion were four ethanolic extracts (E4, oregano flowers; E5, nettle pre-distilled; E6, nettle post-distilled; and E10, yarrow pre-distilled) and one pure compound (P2, rosmarinic acid) (Figure 3D, Table 1). The invasion of C. jejuni 11168 luxS was not affected by these treatments (Supplementary Figure S2D).

It should be noted that none of the treatments achieved the same effects on invasion reduction as did the knock-out mutation of the luxS gene.

\subsection{Correlation Analysis}

The correlations between the QS reductions of the C. jejuni (luminescence signal, logRFU) with the motility (diameter, $\mathrm{mm}$ ), the adhesion to and invasion of INT407 cells ( $\log \mathrm{CFU} / \mathrm{mL})$, and the adhesion to polystyrene surfaces $(\log \mathrm{CFU} / \mathrm{mL})$ after these treatments with essential oils, ethanolic extracts, and pure compounds were examined. Only means for luminescence, colony diameters, and C. jejuni $\log \mathrm{CFU} /$ well were used here. In this way, we determined the correlation of treated cultures without the noise from the error between the experiments.

This analysis showed significant, although moderate, positive correlations of luminescence (i.e., QS) with C. jejuni 11168 motility $(\tau=0.492, p=0.024)$ and adhesion to polystyrene surfaces $(\tau=0.419$, $p=0.008)$. These suggest that the decrease in luminescence, and thus the C. jejuni QS, results in decreased C. jejuni motility on soft agar and adhesion to a polystyrene surface.

These data also showed moderate positive correlation of luminescence and invasion of the INT407 cells $(r=0.394)$, although this remained a trend as statistical significance was not reached $(p=0.068)$. However, this might suggest an influence of the reduced C. jejuni QS on their invasion of these INT407 epithelial cells. 


\section{Discussion}

The LuxS-mediated cell-to-cell signaling system, and thus QS, has an important role in C. jejuni motility, biofilm formation, host colonization, and virulence $[13,15,16,18,19]$.

In the present study, we investigated the modulation of the C. jejuni QS system by (i) knockout mutation of the luxS gene, which is responsible for AI-2 signal production, and (ii) treatments with 20 natural extracts (essential oils, ethanolic extracts, pure compounds). We studied the changes that these modulations might induce in terms of the C. jejuni motility on soft agar, adhesion to polystyrene surfaces, and adhesion to and invasion of human INT407 epithelial cells.

The luxS-deficient mutant showed statistically significant reduction in motility on soft agar (4.7\%), adhesion to polystyrene surfaces (1.47-log reduction), and invasion of INT407 cells (1.10-log reduction) compared to the wild-type C. jejuni. Decreased motility of the luxS-deficient mutant compared to the wild-type was also reported by Elvers and Park [13], Jeon et al. [16], Holmes et al. [17], Quiñones et al. [18], and Plummer et al. [19]. Furthermore, Jeon et al. [16] and Holmes et al. [17] showed that the expression of the flaA gene, which is crucial for flagella-mediated motility, is significantly reduced in the luxS-deficient mutant. Elvers and Park [13] also showed somewhat weaker invasion of Caco-2 cells by the luxS-deficient mutant compared to the wild-type (37.5\% lower) and no effect on adhesion. Quiñones et al. [18], on the other hand, showed significant reduction in adhesion to Leghorn male hepatoma (chicken hepatoma) cells by the luxS mutant compared to the wild-type.

The response of $C$. jejuni 11168 and the knockout mutant C. jejuni 11168 1 luxS to 20 natural formulations, namely six essential oils, 11 ethanolic extracts, and three pure compounds that are often found in plant materials, were compared using the concentration of $0.25 \times$ MIC to ensure they did not influence C. jejuni growth.

The effects of these treatments on the C. jejuni LuxS system were measured with the bioluminescence assay, whereby a reduction in the C. jejuni QS can be inferred from a reduction in the luminescence measured. Significant C. jejuni QS reduction was achieved by the majority of these treatments $(n=15 / 20$; Figure 2). Indeed, a number of these treatments have been previously investigated as QS inhibitors in various organisms, although not in C. jejuni. Juniper [32], lavender [33], cloves [34], thyme [35], and oregano [36] have been reported to show anti-QS activities, which we confirmed here in C. jejuni. Nettle [37] and yarrow [38] had no effects on E. coli QS and Chromobacterium violaceum, respectively, although in the present study they reduced the AI-2 levels (and thus QS) in C. jejuni. In C. violaceum, carvacrol has also been reported to have anti-QS activity [39], although this was not shown here for C. jejuni QS.

Castillo et al. [25] reported that citrus extracts reduced C. jejuni AI-2 activity in this bioluminescence assay, which correlated with its motility and biofilm formation. Furthermore, these citrus extracts reduced C. jejuni invasion of and adhesion to HeLa cells and the expression of the virulence genes cadF and ciaB [26]. On the other hand, Bezek et al. [27] showed good C. jejuni QS reduction with Euodia ruticarpa but no connection to C. jejuni biofilm formation. This indicates high sensitivity of the C. jejuni LuxS system, and thus QS signaling, to outside stressors, such as treatments with natural extracts. However, it does not conclusively confirm the effects of C. jejuni QS inhibition on the other phenotypic changes in C. jejuni.

We showed here that the motility of $C$. jejuni 11168 was significantly reduced by only eight of these 20 treatments, while the motility of C. jejuni 11168 LluxS was not affected by any of them. Interestingly, the greatest effect on motility was achieved by the $S$. rosea (roseroot) ethanolic extract (E8), which also had the largest effect on C. jejuni QS. Indeed, a moderate but significant positive correlation of the luminescence, and thus QS, and C. jejuni 11168 motility was found after these treatments $(\tau=0.492, p=0.024)$. This suggests that treatments with these essential oils, ethanolic extracts, and pure compounds can modulate C. jejuni motility through modulation of the LuxS/QS system. C. jejuni motility is a promising target for reduction of C. jejuni host colonization, as a loss of motility greatly reduces its colonization $[40,41]$. 
Flagella-mediated motility and QS also have important roles in C. jejuni biofilm formation, because non-motile mutants and luxS-deficient mutants have shown weaker biofilm formation compared to the wild-type [42,43]. As a measure of C. jejuni biofilm formation on polystyrene surfaces, we determined here the number of cultivable $C$. jejuni that adhered to the walls of a polystyrene plate. The adhesion of C. jejuni 11168 to the polystyrene surface was significantly reduced by nine of these treatments with essential oils, ethanolic extracts, and pure compounds. The juniper essential oil (EO1) had the strongest effect here, as it reduced C. jejuni adhesion by 1.89-log units (approximately 99\%). The same strong anti-adhesion effect of juniper essential oil (99\% reduction) was also demonstrated by Klančnik et al. [44] using qPCR quantification of C. jejuni adhesion.

Significant moderate positive correlation was found here between luminescence (i.e., QS) and adhesion of $C$. jejuni to polystyrene surfaces after these treatments. This thus indicates that reduced luminescence as AI-2 activity for $C$. jejuni corresponds to reduced adhesion.

The adhesion of the $\operatorname{luxS}$ mutant was lower compared to the wild-type, which again suggests involvement of the LuxS system of C. jejuni in its adhesion to polystyrene surfaces. Consequently, the modulation of the LuxS system results in reduced adhesion of $C$. jejuni.

The influence in the present study of these treatments with essential oils, ethanolic extracts, and pure compounds on C. jejuni invasion of and adhesion to INT407 epithelial cells was tested. Only three of these treatments of C. jejuni 11168 reduced their adhesion to the INT407 cells (E4, oregano,

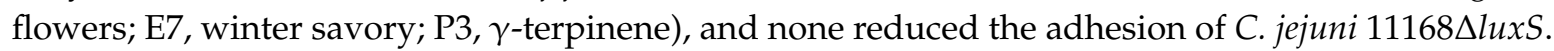
Although the adhesion of $C$. jejuni to the INT407 epithelial cells was generally not affected, the majority of these treatments $(n=15 / 20)$ reduced the C. jejuni 11168 invasion of the INT407 cells. None of the treatments achieved the same level of effect as the knock-out mutation of the luxS gene. This was not surprising, as none of the treatments reduced the C. jejuni QS signal by $100 \%$. The effects of these treatments on C. jejuni 11168 and the absence of effects on C. jejuni 11168 1 luxS show that modulation of the LuxS system by outside stressors can result in changed C. jejuni invasiveness, which in this case was caused by most of these treatments with essential oils, ethanolic extracts, and pure compounds. Furthermore, a moderate, although not statistically significant, positive correlation was shown between C. jejuni AI-2 activity and C. jejuni invasiveness of the INT407 epithelial cells $(r=0.394, p=0.068)$. Lower cell invasiveness of the luxS mutant on Caco-2 cells and no effect on adhesion were previously reported by Elvers and Park [13]. This shows the sensitivity of C. jejuni to the loss or inhibition of the QS system in terms of epithelial cell invasion reduction but not adhesion.

The treatment that showed the greatest C. jejuni QS reduction was the S. rosea (roseroot) ethanolic extract (E8), and this also had the greatest effect on $C$. jejuni motility and was among the best in terms of reduced invasion of INT407 cells and adhesion to polystyrene surfaces by C. jejuni. The roseroot extract is composed of different cyanogenic glycosides, phenylethanoids, procyanidin and catechin derivatives, phenylpropanoids, monoterpene alcohols, and flavonoids [29], and isolated compounds of this extract should be considered in the search for new anti-QS compounds against C. jejuni. Also, rosmarinic acid (P2) was among the weakest in terms of effects on C. jejuni QS, motility, and adhesion to polystyrene surfaces. The moderate positive correlation of the C. jejuni QS signal activity with C. jejuni invasion of epithelial cells, motility, and adhesion to polystyrene surfaces with these treatments and the absence of any effects with the $\Delta l u x S$ mutant indicate a role for the LuxS system in the regulation of these phenotypes. This now defines the LuxS system as an interesting target for the control of C. jejuni and also that such plant-derived substances are potential QS inhibitors and thus potential anti-Campylobacter agents.

\section{Conclusions}

The beneficial effects of used treatments against the wild-type C. jejuni and the lack of effect

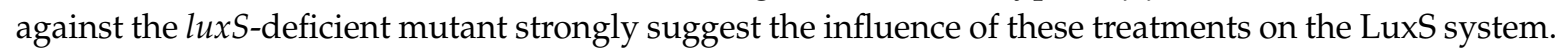
The positive, although moderate, correlation of QS signal reduction with reduced invasion of epithelial cells, motility, and adhesion to polystyrene surfaces links these phenotypic changes. However, as not 
all the changes in QS seen here resulted in the changed phenotypes, other mechanisms of action of such plant extracts remain to be considered before their use for reduction of $C$. jejuni in vivo.

We confirmed here that modulation of the C. jejuni LuxS system with essential oils, ethanolic extracts, and pure compounds can result in reduced C. jejuni invasion of INT407 epithelial cells and motility and adhesion to polystyrene surfaces, and that these phenotypic changes correlate with the measured AI-2 activity. To confirm the anti-QS effects of these treatments, further studies are warranted in C. jejuni. Better quantitative measurement systems and the complementation of the C. jejuni luxS mutant as well as the addition of a pure AI-2 control would further confirm that these natural plant-derived essential oils, ethanolic extracts, and pure compounds act as true QS modulators.

Supplementary Materials: Supplemental materials are available online at http://www.mdpi.com/2076-2607/8/ 1/104/s1, Figure S1: V. harveyi BB170 luminescence production after addition of C. jejuni 11168 and C. jejuni $11168 \Delta \operatorname{luxS}$ cell-free supernatants, and the Mueller Hinton broth control (MHB). Data are means \pm standard deviation of kinetic measurements every $30 \mathrm{~min}$. Dashed box, time point used for further quorum-sensing inhibition measurements (14.5 h); RLU, relative luminescence units, Figure S2: C. jejuni 11168 $\operatorname{luxS}$ (A) motility on $0.4 \%$ agar (colony diameter, $\mathrm{mm}$ ), (B) adhesion on polystyrene (log10 CFU/well), (C) adhesion and (D) invasion of INT407 cells (CFU/mL), without (Control, empty bar) and with treatments with essential oils (EO1-6), ethanolic extracts (E1-11), and pure compounds (P1-3) (full bars). Data are means \pm standard deviations, Table S1: Details of the treatments used in this study, with their corresponding antimicrobial activities, Table S2: C. jejuni 11168 motility (colony diameter in $\mathrm{mm}$ ), adhesion on polystyrene surfaces ( $\log _{10} \mathrm{CFU} /$ well) and invasion of and adhesion to INT407 cells $\left(\log _{10} \mathrm{CFU} / \mathrm{mL}\right)$ in un-treated culture (Control) and in treated cultures. Results are presented as mean \pm standard deviation (SD).

Author Contributions: Conceptualization, K.Š. and S.S.M.; Formal analysis, K.Š. and D.R.; Funding acquisition, S.S.M.; Methodology, K.Š., D.R. and C.X.; Resources, S.S.M.; Validation, K.Š. and D.R.; writing—original draft preparation, K.Š.; writing-review and editing, D.R., C.X. and S.S.M. All authors have read and agree to the published version of the manuscript.

Funding: This project was financed by the Slovenian Research Agency ARRS, programme P4-0116, and the SLO-USA-2018/2019 bilateral projects.

Acknowledgments: The authors would like to thank Franz Bucar, Ivana Turek, Lek Veterina d.o.o., and M. Jeršek sp. for providing the natural extracts, as well as Ajda Kunčič, Mateja Kokalj and Kaja Erzar for help with experimental work. Many thanks also to Q. Zhang and F. Bucar for supervision and support during bilateral project research visits.

Conflicts of Interest: The authors declare that they have no conflict of interest. The funders had no role in the design of the study, in the collection, analyses, or interpretation of data, in the writing of the manuscript, or in the decision to publish the results.

\section{References}

1. Kaakoush, N.O.; Castaño-Rodríguez, N.; Mitchell, H.M.; Man, S.M. Global epidemiology of Campylobacter infection. Clin. Microbiol. Rev. 2015, 28, 687-720. [CrossRef] [PubMed]

2. EFSA and ECDC European Food Safety Authority and European Centre for Disease Prevention and Control. The European Union summary report on trends and sources of zoonoses, zoonotic agents and food-borne outbreaks in 2017. EFSA J. 2018, 16, e05500.

3. Ellyn, P.; Marder, M.P.H. Preliminary incidence and trends of infections with pathogens transmitted commonly through food-Foodborne Diseases Active Surveillance Network, 10 U.S. Sites, 2006-2017. MMWR Morb. Mortal. Wkly. Rep. 2018, 67, 324-328.

4. EFSA and ECDC. European Food Safety Authority; European Centre for Disease Prevention and Control The European Union summary report on antimicrobial resistance in zoonotic and indicator bacteria from humans, animals and food in 2017. EFSA J. 2019, 17, e05598.

5. Helms, M.; Simonsen, J.; Olsen, K.E.P.; Mølbak, K. Adverse health events associated with antimicrobial drug resistance in Campylobacter species: A registry-based cohort study. J. Infect. Dis. 2005, 191, 1050-1055. [CrossRef]

6. BIOHAZ EFSA Panel on Biological Hazards. Scientific Opinion on Campylobacter in broiler meat production: Control options and performance objectives and/or targets at different stages of the food chain. EFSA J. 2011, 9, 2105. 
7. Deep, A.; Chaudhary, U.; Gupta, V. Quorum sensing and bacterial pathogenicity: From molecules to disease. J. Lab. Physicians 2011, 3, 4-11. [CrossRef]

8. Asfour, H.Z. Anti-quorum sensing natural compounds. J. Microsc. Ultrastruct. 2018, 6, 1-10. [CrossRef]

9. Choi, J.; Shin, D.; Ryu, S. Implication of quorum sensing in Salmonella enterica serovar typhimurium virulence: The luxS gene is necessary for expression of genes in pathogenicity island 1. Infect. Immun. 2007, 75, 4885-4890. [CrossRef]

10. Helmy, Y.A.; Deblais, L.; Kassem, I.I.; Kathayat, D.; Rajashekara, G. Novel small molecule modulators of quorum sensing in avian pathogenic Escherichia coli (APEC). Virulence 2018, 9, 1640-1657. [CrossRef]

11. Gorelik, O.; Levy, N.; Shaulov, L.; Yegodayev, K.; Meijler, M.M.; Sal-Man, N. Vibrio cholerae autoinducer-1 enhances the virulence of enteropathogenic Escherichia coli. Sci. Rep. 2019, 9, 4122. [CrossRef] [PubMed]

12. Winstanley, C.; Fothergill, J.L. The role of quorum sensing in chronic cystic fibrosis Pseudomonas aeruginosa infections. FEMS Microbiol. Lett. 2009, 290,1-9. [CrossRef] [PubMed]

13. Elvers, K.T.; Park, S.F. Quorum sensing in Campylobacter jejuni: Detection of a luxS encoded signaling molecule. Microbiol. Read. Engl. 2002, 148, 1475-1481. [CrossRef] [PubMed]

14. Winzer, K.; Hardie, K.R.; Burgess, N.; Doherty, N.; Kirke, D.; Holden, M.T.G.; Linforth, R.; Cornell, K.A.; Taylor, A.J.; Hill, P.J.; et al. LuxS: Its role in central metabolism and the in-vitro synthesis of 4-hydroxy-5-methyl-3(2H)-furanone. Microbiology 2002, 148, 909-922. [CrossRef] [PubMed]

15. Golden, N.J.; Acheson, D.W.K. Identification of motility and autoagglutination Campylobacter jejuni mutants by random transposon mutagenesis. Infect. Immun. 2002, 70, 1761-1771. [CrossRef]

16. Jeon, B.; Itoh, K.; Misawa, N.; Ryu, S. Effects of quorum sensing on flaA transcription and autoagglutination in Campylobacter jejuni. Microbiol. Immunol. 2003, 47, 833-839. [CrossRef]

17. Holmes, K.; Tavender, T.J.; Winzer, K.; Wells, J.M.; Hardie, K.R. AI-2 does not function as a quorum sensing molecule in Campylobacter jejuni during exponential growth in vitro. BMC Microbiol. 2009, 9, 214. [CrossRef]

18. Quiñones, B.; Miller, W.G.; Bates, A.H.; Mandrell, R.E. Autoinducer-2 production in Campylobacter jejuni contributes to chicken colonization. Appl. Environ. Microbiol. 2009, 75, 281-285. [CrossRef]

19. Plummer, P.; Sahin, O.; Burrough, E.; Sippy, R.; Mou, K.; Rabenold, J.; Yaeger, M.; Zhang, Q. Critical role of LuxS in the virulence of Campylobacter jejuni in a guinea pig model of abortion. Infect. Immun. 2012, 80, 585-593. [CrossRef]

20. Adler, L.; Alter, T.; Sharbati, S.; Gölz, G. Phenotypes of Campylobacter jejuni luxS mutants are dependent on strain background, kind of mutation and experimental conditions. PLoS ONE 2014, 9, e104399. [CrossRef]

21. Bjarnsholt, T.; Jensen, P.Ø.; Rasmussen, T.B.; Christophersen, L.; Calum, H.; Hentzer, M.; Hougen, H.-P.; Rygaard, J.; Moser, C.; Eberl, L.; et al. Garlic blocks quorum sensing and promotes rapid clearing of pulmonary Pseudomonas aeruginosa infections. Microbiol. Read. Engl. 2005, 151, 3873-3880. [CrossRef] [PubMed]

22. Harjai, K.; Kumar, R.; Singh, S. Garlic blocks quorum sensing and attenuates the virulence of Pseudomonas aeruginosa. FEMS Immunol. Med. Microbiol. 2010, 58, 161-168. [CrossRef] [PubMed]

23. Jakobsen, T.H.; van Gennip, M.; Phipps, R.K.; Shanmugham, M.S.; Christensen, L.D.; Alhede, M.; Skindersoe, M.E.; Rasmussen, T.B.; Friedrich, K.; Uthe, F.; et al. Ajoene, a sulfur-rich molecule from garlic, inhibits genes controlled by quorum sensing. Antimicrob. Agents Chemother. 2012, 56, 2314-2325. [CrossRef] [PubMed]

24. Peng, L.-Y.; Yuan, M.; Cui, Z.-Q.; Wu, Z.-M.; Yu, Z.-J.; Song, K.; Tang, B.; Fu, B.-D. Rutin inhibits quorum sensing, biofilm formation and virulence genes in avian pathogenic Escherichia coli. Microb. Pathog. 2018, 119, 54-59. [CrossRef]

25. Castillo, S.; Heredia, N.; Arechiga-Carvajal, E.; García, S. Citrus extracts as inhibitors of quorum sensing, biofilm formation and motility of Campylobacter jejuni. Food Biotechnol. 2014, 28, 106-122. [CrossRef]

26. Castillo, S.; Dávila-Aviña, J.; Heredia, N.; Garcia, S. Antioxidant activity and influence of citrus by-product extracts on adherence and invasion of Campylobacter jejuni and on the relative expression of cadF and ciaB. Food Sci. Biotechnol. 2017, 26, 453-459. [CrossRef]

27. Bezek, K.; Kurinčič, M.; Knauder, E.; Klančnik, A.; Raspor, P.; Bucar, F.; Smole Možina, S. Attenuation of adhesion, biofilm formation and quorum sensing of Campylobacter jejuni by Euodia ruticarpa. Phytother. Res. PTR 2016, 30, 1527-1532. [CrossRef] 
28. Bassler, B.L.; Greenberg, E.P.; Stevens, A.M. Cross-species induction of luminescence in the quorum-sensing bacterium Vibrio harveyi. J. Bacteriol. 1997, 179, 4043-4045. [CrossRef]

29. Alperth, F.; Turek, I.; Weiss, S.; Vogt, D.; Bucar, F. Qualitative and quantitative analysis of different Rhodiola rosea rhizome extracts by UHPLC-DAD-ESI-MSn. Sci. Pharm. 2019, 87, 8. [CrossRef]

30. Kovač, J.; Šimunović, K.; Wu, Z.; Klančnik, A.; Bucar, F.; Zhang, Q.; Možina, S.S. Antibiotic resistance modulation and modes of action of (-)- $\alpha$-pinene in Campylobacter jejuni. PLoS ONE 2015, 10, e0122871. [CrossRef]

31. Negretti, N.M.; Konkel, M.E. Methods to Study Campylobacter jejuni Adherence to and Invasion of Host Epithelial Cells. In Campylobacter jejuni: Methods and Protocols; Butcher, J., Stintzi, A., Eds.; Methods in Molecular Biology; Springer New York: New York, NY, USA, 2017; pp. 117-127, ISBN 978-1-4939-6536-6.

32. Kerekes, E.-B.; Deák, É.; Takó, M.; Tserennadmid, R.; Petkovits, T.; Vágvölgyi, C.; Krisch, J. Anti-biofilm forming and anti-quorum sensing activity of selected essential oils and their main components on food-related micro-organisms. J. Appl. Microbiol. 2013, 115, 933-942. [CrossRef] [PubMed]

33. Poli, J.-P.; Guinoiseau, E.; de Rocca Serra, D.; Sutour, S.; Paoli, M.; Tomi, F.; Quilichini, Y.; Berti, L.; Lorenzi, V. Anti-quorum sensing activity of 12 essential oils on Chromobacterium violaceum and specific action of cis-cis-p-menthenolide from Corsican Mentha suaveolens ssp. insularis. Mol. J. Synth. Chem. Nat. Prod. Chem. 2018, 23. [CrossRef] [PubMed]

34. Husain, F.M.; Ahmad, I.; Asif, M.; Tahseen, Q. Influence of clove oil on certain quorum-sensing-regulated functions and biofilm of Pseudomonas aeruginosa and Aeromonas hydrophila. J. Biosci. 2013, 38, 835-844. [CrossRef] [PubMed]

35. Singh, A.; Gupta, R.; Tandon, S.; Pandey, R. Thyme oil reduces biofilm formation and impairs virulence of Xanthomonas oryzae. Front. Microbiol. 2017, 8. [CrossRef]

36. Alvarez, M.V.; Ortega-Ramirez, L.A.; Gutierrez-Pacheco, M.M.; Bernal-Mercado, A.T.; Rodriguez-Garcia, I.; Gonzalez-Aguilar, G.A.; Ponce, A.; del R. Moreira, M.; Roura, S.I.; Ayala-Zavala, J.F. Oregano essential oil-pectin edible films as anti-quorum sensing and food antimicrobial agents. Front. Microbiol. 2014, 5. [CrossRef]

37. Omwenga, E.O.; Hensel, A.; Pereira, S.; Shitandi, A.A.; Goycoolea, F.M. Antiquorum sensing, antibiofilm formation and cytotoxicity activity of commonly used medicinal plants by inhabitants of Borabu sub-county, Nyamira County, Kenya. PLoS ONE 2017, 12. [CrossRef]

38. Tolmacheva, A.A.; Rogozhin, E.A.; Deryabin, D.G. Antibacterial and quorum sensing regulatory activities of some traditional eastern European medicinal plants. Acta Pharm. Zagreb Croat. 2014, 64, 173-186. [CrossRef]

39. Burt, S.A.; Ojo-Fakunle, V.T.A.; Woertman, J.; Veldhuizen, E.J.A. The natural antimicrobial carvacrol inhibits quorum sensing in Chromobacterium violaceum and reduces bacterial biofilm formation at sub-lethal concentrations. PLoS ONE 2014, 9, e93414. [CrossRef]

40. Wassenaar, T.M.; van der Zeijst, B.A.; Ayling, R.; Newell, D.G. Colonization of chicks by motility mutants of Campylobacter jejuni demonstrates the importance of flagellin A expression. J. Gen. Microbiol. 1993, 139 Pt 6, 1171-1175. [CrossRef]

41. Hermans, D.; Van Deun, K.; Martel, A.; Van Immerseel, F.; Messens, W.; Heyndrickx, M.; Haesebrouck, F.; Pasmans, F. Colonization factors of Campylobacter jejuni in the chicken gut. Vet. Res. 2011, 42, 82. [CrossRef]

42. Kalmokoff, M.; Lanthier, P.; Tremblay, T.-L.; Foss, M.; Lau, P.C.; Sanders, G.; Austin, J.; Kelly, J.; Szymanski, C.M. Proteomic analysis of Campylobacter jejuni 11168 biofilms reveals a role for the motility complex in biofilm formation. J. Bacteriol. 2006, 188, 4312-4320. [CrossRef] [PubMed]

43. Reeser, R.J.; Medler, R.T.; Billington, S.J.; Jost, B.H.; Joens, L.A. Characterization of Campylobacter jejuni biofilms under defined growth conditions. Appl. Environ. Microbiol. 2007, 73, 1908-1913. [CrossRef] [PubMed]

44. Klančnik, A.; Zorko, Š.; Toplak, N.; Kovač, M.; Bucar, F.; Jeršek, B.; Smole Možina, S. Antiadhesion activity of juniper (Juniperus communis L.) preparations against Campylobacter jejuni evaluated with PCR-based methods. Phytother. Res. PTR 2018, 32, 542-550. [CrossRef] [PubMed]

(C) 2020 by the authors. Licensee MDPI, Basel, Switzerland. This article is an open access article distributed under the terms and conditions of the Creative Commons Attribution (CC BY) license (http://creativecommons.org/licenses/by/4.0/). 